\title{
„nicht mehr zu erzählen gewusst als \\ unzuverlässige unsichere Gerüchte" Zu \\ den Grenzen unzuverlässigen Erzählens \\ in Uwe Johnsons Mutmassungen über \\ Jakob (1959)
}

\section{Paul Onasch}

Zusammenfassung Im Aufsatz wird die Frage diskutiert, inwiefern der Erzähler in Uwe Johnsons erstveröffentlichtem Roman Mutmassungen über Jakob den Versuch, die Hintergründe zum Tod der Titelfigur Jakob Abs zu rekonstruieren, auf narratologisch unzuverlässige Weise arrangiert. Ausgehend von Simone Elisabeth Langs These, dass „,der Befund zur mimetischen Zuverlässigkeit des Erzählers positiv“ ausfalle, sich das Erzählen im Roman aber ,am Rande des Spektrums der erzählerischen Zuverlässigkeit“" bewege (Lang 2016, S. 223), werden Textstellen untersucht, in denen der Erzähler scheinbar auf das Bewusstsein der zu Beginn der Erzählung bereits verstorbenen Titelfigur zurückgreifen kann. Während es sich bei diesen Passagen eindeutig nicht, wie von Lang angenommen, um unmarkierte Wiederholungen von Figurentext im Erzählerbericht handelt (vgl. Lang 2016, S. 219 f.), bleibt aufgrund des artifiziellen Charakters einiger Bewusstseinswiedergaben offen, inwieweit diese womöglich als szenische Vergegenwärtigung des Erzählers gestaltet sind (vgl. Müller 2005, S. 272). Zweifellos nimmt Johnson damit eine mimetische Unzuverlässigkeit in Kauf, spielt bei genauerer Betrachtung mit ihr (vgl. Lang 2016, S. 223), um den poetologischen Anspruch an einen Erzähler zu demaskieren, eine konsistente (Erzähl-), Wahrheit' präsentieren zu müssen.

Als der gerade einmal 25-jährige Uwe Johnson im Herbst 1959 seinen ersten Roman veröffentlichte, war die Kritik voll des Lobes über das Debüt dieses ,jungen Ostdeutschen, der 1934 in Pommern geboren wurde“ (Wieser 1959,

P. Onasch $(\bowtie)$

Briefedition „Barlach 2020“,

Universität Rostock,

Rostock, Deutschland

E-Mail: paul.onasch@uni-rostock.de 
S. 25). ${ }^{1}$ Marcel Reich-Ranicki, der das Werk zunächst als eine „Provokation und eine unglaubliche Zumutung“ wahrnahm, erkannte in Johnson ,eine ganz große Hoffnung“ (Reich-Ranicki 1959, S. 20). Das ambivalente Urteil Reich-Ranickis gründet in erster Linie auf der von Johnson gewählten Form des Romans: Die Freunde und Bekannten von Jakob Abs, einem Streckendispatcher bei der Deutschen Reichsbahn, stellen nach dessen Tod beim Überqueren der Gleise in den frühen Morgenstunden des 8. November 1956 Überlegungen über die Umstände an, die in Monologen, die kursiv hervorgehoben sind, und Dialogen, die durch Spiegelstriche markiert werden, wiedergegeben werden. Angeordnet, eingeblendet und unterbrochen werden beide Erzählmodi ${ }^{2}$ durch einen nichtdiegetischen bzw. heterodiegetischen Erzähler. ${ }^{3}$ In diesem dritten Erzählmodus, den sog. Erzählerpartien, die den weitaus größten Teil des Textes ausmachen (vgl. Born 1997, S. 75-77), werden Sacherklärungen geliefert, das Geschehen oder das eigene Erzählen kommentiert und Informationen in externen Analepsen bereitgestellt (vgl. Leuchtenberger 2003, S. 141 f.).

In einem Interview mit Arnhelm Neusüss erklärte Johnson, dass das, was die Figuren ,sich da zusammendenken über Jakobs letzte Tage, wie sie gewesen sein könnten, was da war, als sie selbst es nicht sahen - das ist ja unzuverlässig, das bilden die sich ja ein, das sind Interpretationen“" (zit. n. Neusüss 1988 [1961], S. 186). Sie stellen im Sinne des Romantitels Mutmaßungen über den zu Tode gekommenen Jakob an, Mutmaßungen, die ,,immer ungenau und stets unzuverlässig“ sind (Ahl 1960, S. 151). Konsequent erscheint es, den Titel und das damit verbundene Erzählverfahren des Mutmaßens auf den gesamten Roman und somit auch auf den Erzähler und die von ihm verantworteten Erzählerpartien zu über-

\footnotetext{
${ }^{1}$ Nicht verschwiegen bleiben soll dabei, dass der Roman und vor allem Johnson als aus der DDR Übergesiedelter - ein Umstand, der auch in dieser Schweizer Rezension betont wird - in der westdeutschen Kritik mit Attributen wie ,Roman der deutschen Teilung (Siedler) oder ,Dichter der beiden Deutschland' (Blöcker) auf das Thema der deutsch-deutschen Teilung eingegrenzt und für westdeutsche Sichtweisen auf diese vereinnahmt wurde; vgl. hierzu Bond (2009, S. 146 f.).

${ }^{2}$ Vgl. Leuchtenberger (2003, S. 101 f.). Johnson selbst bezeichnete die Erzählmodi als „drei Manieren“ oder „drei Gesten“ des Erzählens (zit. n. Roloff 1988 [1961], S. 177); vgl. auch Johnson (1980, S. 139).

${ }^{3}$ Angesichts der für Johnsons Romane charakteristischen Textinterferenz, der Überlagerung von Erzähler- und Figurentext, wird für die folgende Analyse der Mutmassungen über Jakob auf die stratifikatorische Erzähltheorie Wolf Schmids und deren Terminologie zurückgegriffen. Schmid hebt in Elemente der Narratologie hervor, dass die Binäropposition diegetischer und nichtdiegetischer Erzähler in erster Linie dazu diene, die auf Franz K. Stanzel zurückgehende „traditionelle, aber problematische Dichotomie Ich-Erzähler vs. Er-Erzähler [zu] ersetzen“ (Schmid 2008, S. 89). Im Wesentlichen entspricht Schmids Unterscheidung der Stellung des Erzählers zum erzählten Geschehen - ein zur Diegesis und zur Exegesis oder ein nur zur Exegesis gehörendes Ich - der Differenzierung Gérard Genettes zwischen einem Erzähler, der in der Geschichte, die er erzählt, ,anwesend ist“ (homodiegetisch) oder aber „nicht vorkommt“ (heterodiegetisch) (Genette 1994 [1972], S. 175). Vgl. Schmid (2008, S. 88).
} 
tragen. ${ }^{4}$ Aufgegriffen wurde dieser Gedanke bereits in den ersten Rezensionen zu den Mutmassungen über Jakob. Günter Blöcker (1959, S. V) beschreibt den Erzähler als Vermittlungsinstanz, die die Geschichte der Titelfigur ,nicht als romanhaft präsentierte Folge von Ereignissen ab[spult]“, sondern sie sukzessive entstehen lässt, „,so wie sich Erfahrungen und Wahrnehmungen dem menschlichen Bewußtsein mitteilen: in Stößen, Wellen, Fragmenten, in Erinnerungsschüben, Monologen und Gesprächsfetzen, im Allgemeingewoge des Existentiellen ebenso wie in exakt faßbaren Daten“. Der Erzähler begebe sich in einen Bereich, ,in welchem wie der Titel zu verstehen gibt, allenfalls Mutmaßungen gestattet sind. Was darüber hinausgeht, verletzt bereits die Wahrheit“" (ebd.).

\section{Narratologische Vorüberlegungen zur (Un-) Zuverlässigkeit in den Erzählerpartien des Romans}

Vor dem Hintergrund dieser und ähnlicher Interpretationen der Erzählsituation in den Mutmassungen über Jakob soll der Versuch unternommen werden zu untersuchen, inwieweit die den Monologen und Dialogen des Romans inhärente Unzuverlässigkeit auch auf die Erzählerpartien und damit auf die Erzählinstanz übertragen werden kann.

Ist der Bericht des nichtdiegetischen Erzählers wie die Rede der Figuren geprägt vom Erzählverfahren des Mutmaßens, das Johnsons erstveröffentlichtem Roman zugrunde liegt? Begibt sich der Erzähler, wie von Blöcker angenommen, in einen Bereich des Mutmaßens, in dem er zwangsläufig in den Verdacht gerät, unzuverlässig zu erzählen? ${ }^{5}$

Simone Elisabeth Lang hebt zu Beginn ihrer Untersuchung der Mutmassungen über Jakob im Rahmen ihrer Dissertation zum Unzuverlässigen Erzählen in der Heterodiegese hervor, dass sie den Roman ,zunächst als unzuverlässig erzählt kategorisiert habe“ (Lang 2016, S. 206). Dagegen habe die Analyse des Romans

\footnotetext{
${ }^{4}$ In einem Vortrag, gehalten im Dezember 1975 während einer Vortragsreise im damaligen Jugoslawien, stellte Johnson die poetologische Forderung auf, dass ein Titel „so in Form sein“ müsse, „dass er jedes einzelne Wort des Buches umfassen kann“(Johnson 1988 [1975], S. 55).

${ }^{5}$ Auch Ulrich Krellner, der die Begriffe ,Mutmaßungsstil ‘ und ,Mutmaßungspoetik entschieden zurückweist, weil sie als „experimentelles Formprinzip eine verschwommen spekulierende Haltung des Autors gegenüber den von ihm erfundenen Figuren und Geschichten“ impliziere, bestimmt den Erzähler mit Blick auf seine Erzählperspektive als eingeschränkt und damit nicht allwissend (Krellner 2003, S. 46). Statt auf die Begriffe zu verzichten, scheint es naheliegender, davon abzusehen, sie wie Krellner auf den Autor zu übertragen, und unter dem Mutmaßungsstil ein Formprinzip des Romans mit seinen drei Erzählmodi zu verstehen, wie es Hans Mayer (1992, S. 91 f.) getan hat: „Seit Johnsons Romantitel ist in der deutschen Nachkriegsbelletristik, und nicht nur dort, eine modische Phalanx von Erzählungen und Erzählern einer koketten epischen Skepsis aufmarschiert, die sich viel darauf zugute tat, unscharf zu referieren, dem Leser spannende Unauflösbarkeiten darzubieten, ein bißchen mit dem ,Geheimnis` zu spielen“.
} 
ergeben, dass „der Befund zur mimetischen Zuverlässigkeit des Erzählers positiv“ ausfalle (Lang 2016, S. 223). Lang schränkt aber ein, dass sich der Erzähler des Romans „am Rande des Spektrums der erzählerischen Zuverlässigkeit“ bewege, „bei genauer Betrachtung genau damit zu spielen“ scheine (Lang 2016, S. 223).

Mimetisch zuverlässig ist ein Erzähler gemäß der Begriffsbestimmung Tom Kindts, wenn dessen Aussagen über die fiktive Welt unter der Voraussetzung der Kompositionsstrategie des Werkes ,ausschließlich korrekte und alle relevanten Informationen enthalten" (Kindt 2008, S. 51). Lang (2016, S. 54) präzisiert die Bestimmung mimetischer (Un-)Zuverlässigkeit, indem sie statt des Erzählers den Erzähltext, den Erzählerbericht ins Zentrum ihrer Definition stellt, um Spekulationen über den Wissenshorizont des Erzählers von vornherein auszuschließen: „Ein Erzähltext $\mathrm{T}$ ist genau dann mimetisch unzuverlässig zu nennen, wenn $\mathrm{T}$ (vorübergehend) neben der Annahme $\mathrm{A}_{1}$ über eine fiktive Tatsache implizit oder explizit auch zu $\mathrm{A}_{2-\mathrm{X}}$ autorisiert und dies auf einen Verstoß gegen das Kooperationsprinzip auf der Ebene des Berichts zurückzuführen ist." Sowohl Kindt als auch Lang stellen das von Herbert Paul Grice in Logic and Conversation entwickelte Kooperationsprinzip mit seinen Konversationsmaximen ins Zentrum ihrer Explikationen von (un-)zuverlässigem Erzählen. Für die Bestimmung der (Un-)Zuverlässigkeit des Erzählers bzw. des Erzählerberichts in den Mutmassungen über Jakob gilt daher, darauf zu achten, inwiefern die Aussagen der Erzählinstanz ,alle relevanten Informationen enthalten“, inwieweit sie „so informativ ausfallen, wie es das Erzählinteresse und der Äußerungskontext erfordern und die Erzählsituation erlaubt“" (Kindt 2008, S. 67).

Das dem Roman zugrunde liegende Erzählinteresse spiegelt sich in dessen Titel wider und kann mit den Worten des Autors wie folgt wiedergegeben werden: „Die Hauptfigur ist tot, das Buch ein Versuch, die letzten Tage dieses Mannes zu rekonstruieren. Abschließende Auskünfte sind da nicht möglich, deshalb Mutmaßungen“ (zit. n. Halstenberg 1988 [1969], S. 233). Dieses Erzählinteresse vorausgesetzt, dürfte man von einem zuverlässigen Erzähler erwarten, die Umstände von Jakobs Tod (sukzessive) aufzuklären, sofern - und das ist der entscheidende Punkt der folgenden Untersuchung - es Anhaltspunkte in der Erzählanlage des Romans gibt, aus denen geschlossen werden kann, dass der Erzähler über die entsprechenden Informationen verfügt. Fakt ist, dass die Umstände, unter denen sich das Unglück am Morgen des 8. November 1956 ereignet, bis zum Ende des Romans offen bleiben. Jurij Sacharow erkennt in diesem Umstand eine Konsequenz in der Erzählanlage, weil es nicht dem ,ästhetischen Sinn“ des Romans entspreche, ,eine Diskussion von Jakobs Gedanken kurz vor dem Vorfall“ zu führen oder die Einzelheiten eines ,grausigen Unfalls (oder (Selbst-)Mordes)“ zu schildern (Sacharov 2011, S. 81). Für die Frage nach der (Un-)Zuverlässigkeit des Erzählers ist der ästhetische Sinn aber zunächst zweitrangig. Entscheidend ist, ob und inwieweit sich für den Roman eine ,inkonsistente Beschränkung des Erzählerwissens“ (Leuchtenberger 2003, S. 144) bzw. besser des Erzählerberichts feststellen lässt.

Die ersten beiden Erzählerpartien zeugen von einer Erzählinstanz, die keinen Überblick über das Geschehen hat, sondern für das eigene Erzählen auf Aus- 
sagen der Figuren zurückgreift, sie anreichert und kommentiert. Sacharov (2011, S. 82) hat dieses Erzählprinzip der unmarkierten ,Wiederholungen“ von Figurentext in der Erzählerrede eindrucksvoll an den ersten Seiten des Romans exemplifiziert: „Der erste Satz im Roman, der der Perspektive des Erzählers zuzuordnen ist, wiederholt variierend den Beginn des Dialogs zwischen zwei Bekannten Jakobs, Jöche und Jonas, die seine Todesumstände zu erörtern suchen. "6 Noch deutlicher wird dieses Verfahren bei der Darstellung des Unglücks in der zweiten Erzählerpartie. Der Erzähler konstruiert seinen Bericht auf der Grundlage verschiedener Figurenaussagen, er schildert ,den Bahnknotenpunkt an einem Herbstmorgen (nicht unbedingt am Morgen von Jakobs Tod) und verwendet dabei sowohl Jakobs (und sodann Jonas') Worte über den Nebel, nass verschmierte Gleise, als auch die beobachtende Perspektive des ,F-d-1“ und Jöches Worte über die Erfahrung und Vorsicht Jakobs“" (Sacharov 2011, S. 84). ${ }^{7}$ Auflösen lässt sich durch die Beobachtung Sacharovs auch das scheinbare Paradox, dass der Erzähler an zwei Stellen als vermeintliches Erzähler-Ich auftritt, aber als Figur nicht greifbar wird. ${ }^{8}$ Die Charakterisierung Jakobs und seiner Arbeit als Dispatcher beginnt der Erzähler mit den Worten ,In diesem Herbst war Jakob achtundzwanzig Jahre alt“ und wiederholt sie kurz darauf: „In diesem Herbst nun: wie ich sage“ (MJ, 17 f.). Die inquit-Formel bezieht sich allerdings nicht auf den Erzähler, sondern auf Gesine, die im ersten auf sie zurückgehenden Monolog ihren Vater mit den Worten charakterisiert: „Mein Vater war achtundsechzig Jahre alt in diesem Herbst“ (MJ, 8). ${ }^{9}$ Wenig später berichtet der Erzähler, dass Jakob an einem Abend „,im Ratskeller gesehen [wurde] mit einer Dame“ (MJ, 25), die in der Folge als Sabine, Jakobs Freundin, identifiziert wird. Der Erzähler schließt seinen Bericht vorübergehend mit der Bemerkung, dass Sabine angerufen wurde ,aus dem ganzen Gelände von jungen Männern seines Alters mit dienstlichen Vorwänden, ein Gespräch mit Jakob war lange nicht bemerkt worden, wie ich ja sage“ (MJ, 25 f.), um daraufhin erneut mit dem Erzählen anzusetzen. Die inquit-Formel ,wie ich ja sage" greift den Abschluss der Rede des Taxifahrers auf, der Rohlfs die nächtliche Fahrt von Jakob und Gesine im Taxi berichtet. Hergestellt wird damit eine Verbindung der Beziehungen zwischen Jakob und Sabine bzw. Jakob und Gesine: „Sie hatte die Hände in den Taschen und lehnte an seiner Schulter beim Gehen: als würde er sie tragen ohne sie anzufassen. Wie ich ja sage.“ (MJ, 35).

\footnotetext{
${ }^{6}$ Vgl. Johnson (2017 [1959], S. 7): „Aber Jakob ist immer quer über die Gleise gegangen. / Aber er ist doch immer quer über die Rangiergleise und die Ausfahrt gegangen." Die weiteren Nachweise von Zitaten aus den Mutmassungen über Jakob erfolgen unter der Angabe der Sigle ,MJ' und der Seitenzahl im Text.

${ }^{7}$ Vgl. MJ, 105 f., 201, 203. ,F-d-1‘ ist die Abkürzung für den Fahrdienstleiter, der in einer Betriebsstelle die Zulassung von Zugfahrten und die Zugfolge regelt.

${ }^{8}$ Vgl. etwa Leuchtenberger (2003, S. 141); Krellner (2003, S. 76); zuletzt auch Yamamoto (2015, S. 63).

${ }^{9}$ Sofern der Kursivdruck in Textstellen der Mutmassungen über Jakob wie hier unkommentiert bleibt, handelt es sich um Kursivierungen im Original, die durch Johnson als Hervorhebung des monologischen Erzählmodus vorgenommen wurden.
} 
Ausgehend von dieser ersten exemplarischen Analyse Sacharows lässt sich mit Leuchtenberger (2003, S. 147) festhalten, dass der Erzähler „,mit den Sichtweisen, Perspektiven und Stimmen der Figuren [jongliert], die er wie an einem Mischpult ein- und ausblenden und sich ihrer bedienen kann“. Analog zur Titelfigur nimmt der Erzähler „dispatchende Funktionen“ wahr (Neumann 1993, S. 161). ${ }^{10}$ Sacharov (2011, S. 85 f.) geht noch einen Schritt weiter und bestimmt die Erzählerpartien als ,ein Konvolut von Gedanken und Beobachtungen der handelnden Personen, die vom Erzähler episch bearbeitet und erweitert werden“. Er betont den herausgehobenen Stellenwert der Figuren als Grundlage des Erzählerberichts und erwähnt eher beiläufig die nachträgliche epische Bearbeitung und vor allem die Erweiterung des Figurentextes durch den Erzähler. Ähnlich verhält es sich in der Analyse Langs, die auf der Grundlage der Romaneröffnung und der Relektüre einiger von Leuchtenberger angeführter Textbeispiele zu dem Schluss gelangt, dass der Erzähler eine Art kollektives Gedächtnis der Figuren spiegele und deren Mutmaßungen um meist sachliche Einwendungen erweitere: „Doch es handelt sich keinesfalls um eine durchgängige Übersicht, welche die Erwartungshaltung des Lesers rechtfertigen würde, alle relevanten Informationen zur Rekonstruktion der Ereignisse zu erhalten“ (Lang 2016, S. 219 f.).

Weitgehend unberücksichtigt bleibt die bereits an früherer Stelle erwähnte These Leuchtenbergers, dass die Darstellung der inneren Vorgänge der Figuren durch den Erzähler inkonsistent sei. Angesprochen ist mit dieser These einmal mehr auch die Perspektive, aus der heraus der Erzähler seinen Bericht gestaltet. Unter Zuhilfenahme des Stratifikationsmodells der Erzählperspektive von Wolf Schmid wird deutlich, dass für die Frage der (In-)Konsistenz und damit auch der (Un-)Zuverlässigkeit der Aussagen des Erzählers nicht die perzeptive Ebene der Perspektive entscheidend ist. ${ }^{11}$ Auch wenn sich der Erzähler in den Erzählerpartien systematisch der Perspektive verschiedener Figuren bedient, erzählt er nicht durch deren Prisma bzw. aus ihrem Bewusstsein heraus. Entsprechend sind die Erzählerpartien perzeptiv aus einer manifest narratorialen Perspektive heraus erzählt. Vielmehr gilt es zu untersuchen, ob die Erzählerpartien auf der zeitlichen

\footnotetext{
${ }^{10}$ Neumann geht in seiner Ausführung zur Analogie unnötigerweise noch einen Schritt weiter und setzt den Erzähler mit dem Autor des Romans gleich: „Als Erzähler der Mutmassungen ist der Autor Johnson der Dispatcher Jakob, der im Mittelpunkt des Buches steht. Wie dieser die ZugVorgänge, so steuert Johnson die Erzählvorgänge. Wie dieser über die Abfolge der Züge, verfügt jener über Einsatz und Abbruch der einzelnen Erzählstränge, über deren Koordination. Er verfügt, was den Kosmos des Buches betrifft, über die Zeit, über die erzählte Zeit ebenso wie über die Erzählzeit“" (ebd., S. 161 f.).

${ }^{11}$ Zurückgehend auf Boris A. Uspenskijs Poetik der Komposition differenziert Schmid (2008, S. 130-137) die Erzählperspektive in die fünf Parameter Raum, Ideologie, Zeit, Sprache und Perzeption. Mit der Perzeption erfasst Schmid (2008, S. 136) „das Prisma, durch das das Geschehen wahrgenommen wird. Auf die perzeptive Perspektive zielen Fragen wie ,Mit wessen Augen blickt der Erzähler auf die Welt?` oder ,Wer ist für die Auswahl dieser und nicht anderer Momente des Geschehens für die Geschichte verantwortlich?“
} 
Ebene konsistent erzählt sind. ${ }^{12}$ Die für die Einhaltung des Kooperationsprinzips nötige Annahme, dass der Erzähler über den Tod Jakobs alles Relevante berichtet, was er zu berichten weiß, setzt voraus, dass er über das konkrete Geschehen um die Titelfigur retrospektiv nur das zu erzählen imstande ist, was ihm durch die Rekonstruktionsversuche der im Roman handelnden Figuren zugänglich ist. Wenn der Erzähler die Gedanken und Gefühle des bereits verstorbenen Jakob referiert, müsste diese für ein zuverlässiges Erzählen entweder auf die Perspektive (einer) der Figuren zurückzuführen oder als Mutmaßung des Erzählers ausgewiesen sein. Inkonsistent im Sinne des Kooperationsprinzips wäre es dagegen, wenn der Erzähler innerhalb seines Berichts vorgäbe, über einen (uneingeschränkten) Zugriff auf die Gedanken und Gefühle des verstorbenen Jakob zu verfügen, diese in den Momenten vor dem Tod Jakobs aber verschweigt bzw. dessen Perspektive auf die Ereignisse ausspart. ${ }^{13}$ Für die Überprüfung dieser Überlegungen und der ihr zugrunde liegenden Frage nach der (Un-)Zuverlässigkeit des Erzählers in den Mutmassungen über Jakob werden im Folgenden ausgewählte Bewusstseinswiedergaben Jakobs durch den Erzähler analysiert.

\section{Wiederholendes Erzählen oder szenische Vergegenwärtigung? Zu ausgewählten Bewusstseinswiedergaben des Erzählers}

Innerhalb des zweiten (Erzähl-)Abschnitts im ersten Kapitel, ${ }^{14}$ der insgesamt zehnten Erzählerpartie des Romans, wird anhand exemplarischer Sequenzen und aus einer manifest narratorialen Perspektive Jakobs Arbeits- und Privatleben beschrieben. Der Erzähler, der hierfür auf die Recherchen des SSD-Hauptmanns Rohlfs zurückgreift und dessen investigative Recherche narrativ nachzubilden versucht, nähert sich mittels eines „Zoom-Ins“ (Jäger 2009, S. 218) dem Privatleben des Eisenbahndispatchers an: „An einem Abend mitten in der Woche wurde Jakob im Ratskeller gesehen mit einer Dame.“ (MJ, 25) Ausgehend von dieser Beobachterperspektive ,enthüllt“ der Erzähler sukzessive, dass die Dame „,[b]ei

\footnotetext{
${ }^{12} \mathrm{Nicht}$ weiter beachtet werden muss in diesem Zusammenhang die räumliche Ebene, da der (nichtdiegetische) Erzähler nicht Teil der erzählten Welt (Diegesis) ist.

${ }^{13}$ Nach James Phelan und Mary Patricia Martin läge in diesem Fall eine Form des underreportings vor: „Underreporting, which Genette calls paralipsis, occurs when the narrator tells us less than s/he knows. When Stevens reports that he has denied working for Lord Darlington but defers telling us about Lord Darlington's disgrace, he is underreporting" (Phelan und Martin 1999, S. 95). Vgl. hierzu auch Kindt (2008, S. 49).

${ }^{14}$ Die in fünf Kapitel untergliederten Mutmassungen über Jakob werden auf einer zweiten makrostrukturellen Ebene in (Erzähl-)Abschnitte untergliedert, die durch die typographische Hervorhebung der ersten drei Wörter mittels Kapitälchen markiert werden. Vgl. hierzu Leuchtenberger (2003, S. 110-115), die terminologisch nicht zwischen ,Kapitel ‘ und ,(Erzähl-) Abschnitten', sondern zwischen ,Büchern“ und ,Kapiteln“ unterscheidet.
} 
einem Vergleich mit den allmählich vorhandenen Bildern [...] als Sabine namhaft“ (MJ, 26) wurde. Innerhalb der Montage verschiedener (Figuren-)Perspektiven, auf die Rohlfs durch Befragungen zurückzugreifen vermag, zoomt der Erzähler bis hin zur Wiedergabe der Gedanken und Gefühle der Titelfigur: „Jakob sass neben Sabine an einem Pfeiler in dem niedrigen ausgemalten Gewölbe und legte ihr das Essen vor und bediente sie in allem (fühlte ihre Blicke in seinem Gesicht und fühlte den grossen trägen wehen Überdruss, den er ihretwegen gern geleugnet hätte. Das Gesicht ist gehorsam und lächelt, aber bewege dich nur so wenig wie immer)" (MJ, 26). Die Beobachterperspektive verlassend, beschreibt der Erzähler innerhalb der Klammer die Gefühle Jakobs zunächst aus der narratorialen Perspektive und damit als Erzählertext. Weiter gesteigert wird die Unmittelbarkeit durch die Integration von Figurentext mittels direkt wiedergegebener Rede im zweiten Satz. Die Szene wird schrittweise vergegenwärtigt, die epische Distanz sukzessive eingeholt, um schließlich den Eindruck zu erzeugen, einen „Bewußtseinssplitter“ (Jäger 2009, S. 218) der Titelfigur wiederzugeben. Vor dem Hintergrund von Sacharovs Beobachtung eines ,wiederholenden Erzählens ' in den Mutmassungen über Jakob und der daraus abgeleiteten mimetischen Zuverlässigkeit des Erzählers durch Lang müsste sich die Introspektion bei näherer Untersuchung auf Aussagen oder Gedanken einer anderen Figur zurückverfolgen lassen. Alternativ wäre die mimetische Zuverlässigkeit gewahrt, wenn die Bewusstseinswiedergabe des zum Zeitpunkt des Erzählens bereits verstorbenen Jakob als szenische Vergegenwärtigung der Phantasie des Erzählers entspränge mit dem Ziel der „Eröffnung eines autonomen Erinnerungsraums“ (Neumann 1993, S. 154) für die Figur. ${ }^{15}$ Beiden Möglichkeiten wird im Folgenden nachgegangen, angefangen mit einer auf Sacharovs Beobachtungen basierenden Untersuchung zum wiederholenden Erzählen in Johnsons erstveröffentlichtem Roman.

Die metonymische Beschreibung des Gesichts von Sabine als eines, das „gehorsam [ist] und lächelt"“, wird im Romantext kein zweites Mal aufgegriffen. Auch der Adversativsatz ,aber bewege dich nur so wenig wie immer“ findet sich an keiner weiteren Stelle im Roman. Einzig der adverbiale Gebrauch von ,gehorsam" wird in einem späteren Abschnitt erneut aufgegriffen. In einem Monolog lässt der Assistent am Anglistischen Seminar der Humboldt Universität zu Berlin, Jonas Blach, die erste, zufällige Begegnung mit Gesine Cresspahl, seiner späteren Geliebten und der Ziehschwester Jakobs, auf einer Straße ,in einem Berliner Vorort abends“ (MJ, 88) Revue passieren. Er erinnert sich an ihre sonderbare Frage ,Which feature is it? “ und seine Reaktion: „Welcher Film war es: dachte ich gehorsam. “ (MJ, 88) Ohne die Wiederholung des Adverbs überstrapazieren zu wollen, lässt sich über den jeweiligen Kontext der Szenen hinaus das Wiederaufgreifen des Lexems als Prolepse auf das Schicksal der Beziehung zwischen Gesine und Jonas lesen - wie sich Jakob von (der gehorsam blickenden) Sabine trennt, findet auch Gesine in (dem gehorsam denkenden) Jonas nicht ihr

${ }^{15}$ Zur szenischen Vergegenwärtigung in den Mutmassungen über Jakob vgl. Müller (2005, S. 272). 
Liebesglück. Folglich ließe sich der Bewusstseinssplitter als szenische Vergegenwärtigung des Erzählers deuten. Als eine solche markiert ist der Textabschnitt indes nicht.

Wie in diesem Fall gibt es auch in einer zweiten Szene keinen Hinweis darauf, dass der Erzähler für die Darstellung von Jakobs Gedanken und Gefühlen auf die Perspektive einer anderen als der Titelfigur zurückgreift. Das erste Gespräch zwischen Jakob und Rohlfs findet in der Dienststelle des SSD-Hauptmanns, in einem „fensterlosen Würfel von Zimmer“ (MJ, 37) statt, nachdem der Dispatcher auf der Straße angehalten und aufgefordert wurde, in den Pobjeda von Rohlfs zu steigen. Bereits seit Jakobs Ablösung durch seinen Eisenbahnerkollegen Wolfgang Bartsch nähert sich der Erzähler schrittweise der Perspektive der Titelfigur an. ${ }^{16}$ Auf eine erste Introspektion greift er zurück, um die Diskrepanz zwischen Bartschs Anstellung als Dispatcher und seinem Bildungsweg, der in einer externen Analepse erzählt wird, hervorzuheben: „Wolfgang war in der Oberschule ausgebildet und hatte ein Studium hinter sich gebracht. Das war schon vier oder fünf Jahre her, aber unwillkürlich behielt Jakob es in seinem Gedächtnis als sei es bedeutsam für die Bitterkeit, die Wolfgang von da oder anders überstehen mochte." (MJ, 35) Für die Darstellung von Jakobs Weg von seiner Dienststelle zur Bushaltestelle schildert der Erzähler an zwei weiteren Stellen dessen Erinnerungen, bevor er ihn im Wagen des SSD-Hauptmanns „,die Müdigkeit wie ein Gewicht in seinen Gliedern und in seinen Gedanken“ fühlen lässt: „Einmal erschrak er weil er an keinen Menschen eigens dachte, das wurde geringes Unbehagen, er vergass es.“ (MJ, 36) Auch während des Gesprächs mit Rohlfs, dessen Inhalt durch den Erzähler ausgespart bleibt, werden „Einblicke in sein Bewusstsein“ (Jäger 2009, S. 216) gewährt:

Jakob betrachtete nun Herrn Fabians Gesicht. (Er stellte sich mit diesem Namen vor für Jakob in leichtem Verbeugen.) Dessen Gesicht war eines von denen, mit denen Jakob nichts zu tun hatte von sich aus und die er nicht einsah bei zufälligen blickweisen Begegnungen auf der Strasse oder anderswo, und dies war eins von denen, die er nicht gleich für eine sonderliche Art oder den Beruf genommen noch überhaupt bedacht hätte. Das Gesicht wandte sich ihm zu mit aufmerksamen sanftbraunen Augen, die aber ausser Jakob noch andere Dinge abwogen, und Jakob dachte von dieser breiten buckligen Stirn und diesen Mundwinkeln in einem viereckigen fleischigen Gesicht dass sie sich wenig würden nehmen lassen wollen von ihrem vermeintlichen Recht. (MJ, 37)

In den folgenden Dialogpassagen mutmaßen Jonas und Jöche über den Inhalt des Gesprächs; den Spekulationen werden Rohlfs Erinnerungen an die Unter-

\footnotetext{
${ }^{16} \mathrm{MJ}, 34$ f.: „Wolfgang Bartsch [kam] mit seinem Schlüssel zu Jakob in den Raum um ihn abzulösen [...] und zog einen Merkstrich unter die von Jakob verwaltete Zeit. Er hatte nicht einmal blickweise Antwort erwartet, und Jakob sah sich nicht eigens um. Er schüttete die Asche und die unzähligen Zündhölzer aus der Schale und rief über das mittlere Telefon auf der rechten Seite den Amtsdispatcher an und meldete sich also ab. [...] Er nickte noch, als Jakob Mahlzeit gesagt hatte; vom Flur her war die heftige nervöse Art seines Redens leise zu hören. Jakob zog seinen Schlüssel ab und ging unter lose übergehängtem Mantel und mit der Tasche am Arm den Flur hinunter dem gemischten Zwielicht des Treppenhauses entgegen.“
} 
redung in Monologform gegenübergestellt (vgl. MJ, 37-43). Den Abschluss des (Erzähl-)Abschnitts bildet eine Erzählerpartie, in der erneut Jakobs Gedanken und Gefühle geschildert werden: „Jakob war nicht unruhig. Er fühlte sich unbeweglich zwischen Lampenschirm und Polsterlehne und Tischrand [...]. Jakob dachte: Er ist einer der besten Dispatcher, sonst weiss ich nichts von ihm." (MJ, 46 f.)

Die in den Erzählerpartien wiedergegebenen Einblicke in das Bewusstsein der Titelfigur, ob die narratorial perspektivierte Darstellung der Vernehmungsszene oder die direkt zitierten Gedanken der Figur, lassen sich auf keine zum Erzählzeitpunkt noch lebende Figur zurückverfolgen: „Der Wahrnehmungsstandpunkt [...] ist unstrittig [der] des Jakob Abs“ (Jäger 2009, S. 217). Der Erzähler suggeriert innerhalb dieser Erzählerpartien, auf das Bewusstsein der Titelfigur zugreifen zu können. Langs These, wonach der Erzähler der Mutmassungen über Jakob zuverlässig erzählt, weil er die Einblicke in das Bewusstsein Jakobs aus Monologen und Dialogen der nach Jakobs Tod mutmaßenden Freunde und Bekannten zusammensetzt, hält einer kritischen Überprüfung nicht stand. Selbst in Fällen, in denen sich Teile des aus Jakobs Bewusstsein Erzählten auf eine andere Figur zurückverfolgen lassen, können nicht uneingeschränkt alle in der entsprechenden Passage wiedergegebenen Gedanken und Gefühle der Titelfigur dieser anderen Figur zugeschrieben werden.

Um die Frage nach der (Un-)Zuverlässigkeit des Erzählers in den Mutmassungen über Jakob abschließend zu beantworten, soll in einem zweiten Schritt untersucht werden, ob sich innerhalb der Erzählerpartien Anhaltspunkte dafür finden, die Wiedergabe von Gedanken und Gefühlen des verstorbenen Jakob durch den Erzähler als simulierte Gedankenrede, als szenische Vergegenwärtigung zu begreifen. Hierzu wird zunächst eine Sequenz analysiert, in der der Erzählerbericht als Mutmaßung markiert ist. ${ }^{17}$

Der vierte (Erzähl-)Abschnitt des zweiten Kapitels, der ausschließlich aus einer Erzählerpartie besteht, beginnt bereits mit einer Rekurrenz auf das Bewusstsein Jakobs: „FAST UNABLÄSSIG AN den beiden folgenden Tagen betrachtete Jakob den jungen Mann aus den Städten Berlin in seinen Gedanken.“ (MJ, 103; Kapitälchen im Original) Von Beginn an weist der Erzähler aus, dass die folgenden Ausführungen zu Jonas, der in Gesines Vaterhaus in Jerichow einen staatskritischen Essay verfasst, maßgeblich auf der Perspektive Jakobs beruhen. Verstärkt wird dieser erste Hinweis durch weitere Introspektionen, die schließlich in eine Passage münden, die ,alle Formen der Bewusstseinswiedergabe vom Bewusstseinsbericht über das Gedankenzitat bis hin zur erlebten Rede“ (Jäger 2009, S. 217) enthält:

Und welchen Zweck verfolge ich? dachte Jakob gelassen spöttisch vor seinem Bildblatt; er fand seine Stimmung selbst wunderlich. Verfolgte Jonas den Zweck die Welt zu bereichern um seine Weise sie anzusehen? vielleicht. Es war nicht sein Beruf ,philosophisch über das Subjekt" zu reden, es hatte ihn überkommen, nicht wahr? und von dieser Redezeit her gesehen waren alle Überdrüsse und Enttäuschungen der albern verwarteten Jahre gerechtfertigt, ja? Wenn einer nun immer den Grundsatz macht aus seinen

\footnotetext{
${ }^{17}$ Vgl. hierzu Leuchtenberger (2003, S. 142 f.) und auch Fahlke (1982, S. 158).
} 
neuesten Umständen: dachte Jakob, er dachte aber nicht an Herrn Rohlfs. Ihm war eingefallen dass er Peter Zahn einen Anruf versprochen hatte, und es war ihm unbehaglich dass er diesen Anruf zu vergessen vorhatte. (MJ, 109)

Den Ausgangspunkt der ausführlichen Gedankenwiedergabe bildet ein Gedankenzitat Jakobs, mit dem er auf Jonas' Bericht über eine wissenschaftliche Versammlung reagiert, „der Einzelne verfolg[e] seinen eigenen Zweck in jedem Tun“ (MJ, 109). In der Folge wechseln sich Bewusstseinsberichte und erlebte bzw. direkte Gedankenrede ab. Auch die weiteren Überlegungen Jakobs zu Jonas' gesellschaftlicher Rolle, das Gesehene, Gelesene und Gesprochene zum real existierenden Sozialismus niederzuschreiben und damit an einem Reformprozess beteiligt sein zu wollen, enthalten zwei weitere Hinweise des Erzählers auf die Wiedergabe von Gedanken und Erinnerungen der Titelfigur. ${ }^{18}$ Die Sequenz endet neuerlich mit einem Gedankenzitat, einem ,stream of consciousness-Fragment" (Jäger 2009, S. 217): „Denn Cresspahl wollte er [= Jonas, P.O.] immerhin eine andere (seine) Meinung beibringen über seine Tochter; die war aber in die Ferne gereist. Und Jonas nach Jerichow. Und meine Mutter in die Flüchtlingsbaracken von Westberlin mit der Eisenbahn, und ich sorge dafür dass sie alle sicher und pünktlich kommen wohin sie wollen.“ (MJ, 110)

Zunächst lässt sich unter der Maßgabe des , wiederholenden Erzählens" in den Mutmassungen über Jakob festhalten, dass sich die vom Erzähler wiedergegebenen Formen der Bewusstseinswiedergabe nicht auf bereits zuvor und an späterer Stelle wiedergegebene Monolog- und Dialogpassagen im Roman zurückführen lassen. Innerhalb des (Erzähl-)Abschnitts fällt darüber hinaus der Einsatz von Modaladverbien und Konjunktivformen, Fragen und Relativierungsformeln auf: „Denn war Jonas nicht sofort verreist? [...] und nun hatte er wahrscheinlich wieder den Blick seines Chefs gesucht in der Gruppe [...]. Und der alte Mann würde genickt haben abwesend in anderen Gedanken mit seinem vorsichtigen wissenschaftlich zerschliffenen Gesicht: Arbeitsurlaub. Nennen wir es so. Nennen wir es so [...]. Und mochte vor Cresspahl vielleicht manchmal des beunruhigten Gewissens sich entsinnen“ (MJ, 109 f.; Hervorhebungen P.O.). Jakobs Überlegungen werden durch die lexikalischen und grammatischen Relativierungsformen als unzuverlässig markiert. Betrachtet man die Szene zunächst isoliert, gibt es keine Hinweise darauf, die Existenz der Gedanken Jakobs in Zweifel zu ziehen. Vielmehr werden sie aufgrund des begrenzten Wissenshorizontes der Figur als Mutmaßungen ausgewiesen. Erweitert man den Analysegegenstand jedoch auf die Wiedergabe von Jakobs Bewusstsein nach dessen Gespräch mit Kasch, einem weiteren Kollegen (vgl. MJ, 104-106), fällt ein Hinweis des Erzählers auf, mit dem er markiert, über Jakobs Gedanken und Gefühle zu mutmaßen: „Jakob hätte gedacht Er soll mir vom Leibe bleiben vom Acker gehen, nicht mehr“ (MJ, 106; Hervorhebung P.O.). In der Folge werden Jakobs Gedanken um verschiedene

\footnotetext{
${ }^{18}$ Vgl. MJ, 109: „Gelesenhaben: dachte Jakob, Geredethaben: er erinnerte sich“; ,wie Jakob überrascht gewahr wurde“.
} 
Rede- und Gedankensplitter anderer Figuren, ${ }^{19}$ Wertungen und Korrekturen des Erzählers ${ }^{20}$ ergänzt mit dem Ergebnis einer polyperspektivischen Collage, in der eine klare Zuordnung von Worten und Gedanken bisweilen schwierig, wenn nicht unmöglich ist. ${ }^{21}$ Dennoch finden sich innerhalb der Erzählerpartien keine direkten Hinweise darauf, die etwa durch inquit-Formeln mit einem verbum credendi oder den Gebrauch des Personalpronomens ,ich“ als Gedanken und Gefühle der Titelfigur gekennzeichneten Passagen als Mutmaßungen des Erzählers anzusehen. Vielmehr liefert der Erzähler vereinzelt Hinweise, die es dem Leser erlauben, die in die Erzählerpartien integrierten Introspektionen auf Jakob als szenische Vergegenwärtigung zu interpretieren. Wie indiziell eine solche Deutung ausfallen kann, soll an einem letzten Beispiel vorgeführt werden.

Im vierten Abschnitt des ersten Kapitels wird Jakobs Freund, der Lokomotivführer Jöche, zunächst vorgestellt, bevor sich beide in einer „Gastwirtschaft an der Industriestrasse“ (MJ, 49) treffen. Jöche teilt Jakob mit, dass ihm das Gerücht zu Ohren gekommen sei, ,dass Cresspahl aus Jerichow zum Westen eingegangen sei“ (MJ, 50), woraufhin dieser wider Erwarten wenig überrascht reagiert. Stattdessen zweifelt Jakob das Gerücht an: „,Was die Leute reden“ sagte Jakob, er dachte wirklich: Dann haben Sie Cresspahl bloss einsteigen nicht aussteigen sehen, nun machen sie eine Geschichte für Cresspahl als ob die Dinge wären wie einer sie ansieht.“ (MJ, 51) Die Formulierung „wie einer sie ansieht“ findet sich ein weiteres Mal in einem Monolog von Rohlfs, der einen Assistenten über die angezeigte Arbeitsweise des Ministeriums für Staatssicherheit belehrt:

Sie haben schon die Gewohnheit nur das allenfalls Straffällige zu sehen, damit erfahren Sie nichts. Sie wissen mehr von einem Menschen wenn Sie rauskriegen wie er seine Kinder behandelt und ob er seinen Freunden gefällig ist für Gegendienste oder bloss so und wie er die Häuser ansieht, die wir gelegentlich aufbauen, tun wir doch: wie einer sie ansieht der Architektur nach, verstehen Sie. Zwischen Staatsbürger und Staatsfeind darf man nicht eine Grenze ziehen vorher. Jedermann ist eine Möglichkeit. Ja? (MJ, 66).

\footnotetext{
${ }^{19} \mathrm{Vgl}$. etwa MJ, 108: „wenn Jonas damit erklären wollte dass er nicht fünf sondern zwanzig Minuten lang gesprochen hatte und nicht eine Zurückweisung sondern Vorschläge. (Und ich fand doch den ganzen Betrieb albern. Ich war einer von den Jüngsten im Saal, Urteil der Zuhörer, eigenes: ich bin schon wieder zu alt als dass ich mir noch erstaunliche Leistungen für die Zukunft gutschreiben könnte; ich bin nicht überdurchschnittlich begabt. Stelle mich hin, mache Ansprüche.) So mochte er reden vor Cresspahl (mein Vater ist ein Turm mit kurzen grauen Scheitelhaaren)“.

${ }^{20} \mathrm{Vgl}$. etwa MJ, 106: „Er hatte auch nicht vergessen dass Jonas nach Gesine gefragt hatte als sei er lange ohne Nachricht von ihr. Wirklich war Herr Dr. Blach in der vorigen Woche täglich unter der Erde auf die andere Seite Berlins gefahren.“

${ }^{21}$ Entsprechend konstatiert auch Jäger zum abschließenden Teil der Gedankenwiedergabe Jakobs (vgl. MJ, 110), dass ,zunächst unklar ist, ob der eigenwillige Rededuktus der Stimme des Erzählers zugerechnet werden muss, oder welchen Status die in Klammern eingefügten deiktischen Präzisierungen haben“" (Jäger 2009, S. 217).
} 
Der parallele Gebrauch der Wendung hebt in beiden Fällen die Reflexionsfähigkeit der jeweiligen Figuren hervor. Jakob und Rohlfs werden, obwohl sie gegenüber Gesine - dem Ziel von Rohlfs Auftrag ,Taube auf dem Dach“ (MJ, 10), die inzwischen in der Bundesrepublik lebende Dolmetscherin für den Staatssicherheitsdienst zu gewinnen - ganz unterschiedliche Interessen verfolgen und auch in ihrer politischen Haltung durchaus voneinander abweichende Ansichten haben, in eine gewisse Nähe gerückt. Rohlfs glaubt diese Nähe bereits im ersten Gespräch zwischen beiden zu erkennen, wenn er bemerkt, ,irgend etwas an seiner Art zu denken kam mir untergründig bei, allmählich verfiel ich auf Ähnlichkeit" (MJ, 39). Insofern lässt sich der wiederholte Gebrauch der Wendung auf eine immanente Poetik der Mutmassungen über Jakob zurückführen: Zwei Figuren werden trotz ihrer divergierenden Interessen, trotz ihrer Opposition als Staatsbürger und Staatsvertreter in ihrem Denken zusammengeführt, um vorzuführen, dass die Dinge nicht sind, „wie einer sie ansieht“ (Hervorhebung P.O.). Keinen hinreichenden Anhaltspunkt bietet das parallele Vorkommen für die Annahme, dass die vom Erzähler wiedergegebene Gedankenrede Jakobs auf Rohlfs Perspektive oder die anderer Figuren zurückgeführt werden könne. Die wörtliche Integration der Figurenrede von Rohlfs verleiht der Gedankenrede Jakobs aber einen artifiziellen Charakter und deutet darauf hin, dass sie einer szenischen Vergegenwärtigung des Erzählers entspringt und nicht dem Zugriff der Erzählinstanz auf das Bewusstsein der Titelfigur.

Fasst man die Ergebnisse der Analyse zusammen, lässt sich mit Lang konstatieren, dass der Erzähler der Mutmassungen über Jakob mit der mimetischen Zuverlässigkeit spielt (vgl. Lang 2016, S. 223). Die wiederholt in die Erzählerpartien integrierten, mehr oder weniger verborgenen Hinweise, die die Wiedergabe der Gedanken und Gefühle Jakobs als Mutmaßungen, als szenische Vergegenwärtigungen markieren, sprechen dafür, das Erzählen des nichtdiegetischen Erzählers als mimetisch zuverlässig zu bestimmen. Der bisweilen erzeugte Eindruck, der Erzähler könne (uneingeschränkt) auf die Gedanken und Gefühle der zum Zeitpunkt des Erzählens bereits verstorbenen Titelfigur zugreifen, werden wiederholt gebrochen. Entsprechend kann der Leser im Sinne des Kooperationsprinzips nicht erwarten, vom Erzähler über die Ursachen für Jakobs Tod aufgeklärt zu werden.

\section{Das Spiel mit der mimetischen (Un-)Zuverlässigkeit und die ,schwierige Suche nach der Wahrheit"6}

Das ,Spiel mit der (Un-)Zuverlässigkeit‘ in den Mutmassungen über Jakob lässt sich auf die Poetik des Romans zurückführen, konkret auf den Versuch, auf die „Manieren der Allwissenheit“, den ,göttergleichen Überblick eines Balzac“ zu verzichten (Johnson 1975 [1961], S. 20). In seinem 1961 entstandenen Aufsatz Berliner Stadtbahn (veraltet), einem der zentralen poetologischen Texte 
Johnsons, ${ }^{22}$ in den, wie noch zu zeigen sein wird, Überlegungen eingegangen sind, die bereits den Entstehungsprozess der Mutmassungen über Jakob maßgeblich beeinflussten, erklärt Johnson die Allwissenheit eines Erzählers wie in den Romanen Honoré de Balzacs als „bewunderswert[e]“, aber unzeitgemäße Form realistischen Schreibens:

Wenn der Verfasser seinen Text erst erfinden und montieren muß: wie kann er dann auf hohem Stuhl über dem Spielfeld hocken wie ein Schiedsrichter beim Tennis, alle Regeln wissen, die Personen sowohl kennen als auch fehlerlos beobachten, zu beliebiger Zeit souverän eingreifen und sogar den Platz tauschen mit einer seiner Personen und noch in sie blicken, wie er sogar selbst sich doch selten bekannt wird. Der Verfasser sollte zugeben, daß er erfunden hat, was er vorbringt, er sollte nicht verschweigen, daß seine Informationen lückenhaft sind und ungenau. Denn er verlangt Geld für was er anbietet. Dies eingestehen kann er, indem er etwa die schwierige Suche nach der Wahrheit ausdrücklich vorführt, indem er seine Auffassung des Geschehens mit der seiner Person vergleicht und relativiert, indem er ausläßt, was er nicht wissen kann, indem er nicht für reine Kunst ausgibt, was noch eine Art der Wahrheitsfindung ist. (Johnson 1975 [1961], S. 20 f.)

Vor dem Hintergrund einer bipolaren Weltordnung - in Johnsons erstveröffentlichtem Roman durch das Nebeneinanderstellen von ungarischem Volksaufstand und Suez-Krise abgebildet und zusammengeführt - erhebt Johnson die narrative Annäherung an die ,Wahrheit' zu seinem obersten poetologischen (Form-)Prinzip. Damit einher geht aber nicht das Primat der Form gegenüber dem Inhalt bzw. der Geschichte. Vielmehr dürfe, so Johnson vierzehn Jahre später, ,[d]as Problem von Form und Inhalt $[\ldots]$ nicht mehr sichtbar sein. Die Geschichte muss sich die Form auf den Leib gezogen haben. Die Form hat lediglich die Aufgabe, die Geschichte unbeschädigt zur Welt zu bringen. Sie darf vom Inhalt nicht mehr ablösbar sein“ (Johnson 1988 [1975], S. 60). Dass diese Maximen bereits für die Entstehung der Mutmassungen über Jakob den poetologischen Hintergrund bildeten, verdeutlicht der mehrfach von Johnson dargestellte Prozess der Suche nach einer passenden Form für seinen zweiten Roman, um die Suche nach der ,Wahrheit' in einer der Geschichte angemessenen Weise zu präsentieren:

Ich habe zuerst versucht, die ganze Geschichte aus dem Blickwinkel eines Erzählers zu schreiben. Dabei entstanden stilistische Schwierigkeiten, da in einem gewissen Sinn der Erzähler die Meinungen und Absichten eines Hauptmanns des Staatssicherheitsdienstes wohlwollend hätte reproduzieren müssen. Da ich aber diese Perspektive nicht zur gleichen Zeit einnehmen konnte, zu der ich aus anderen Blickwinkeln zu sprechen hatte, war ich gezwungen, Rohlfs für sich selbst sprechen zu lassen. (Roloff 1988 [1961], S. 178)

\footnotetext{
${ }^{22}$ Den aus einem Vortrag auf der Forth Annual McGregor Detroit Adventure Conference im April 1961 hervorgegangenen Aufsatz veröffentlichte Johnson wenige Tage vor dem Bau der Berliner Mauer in deutscher Übersetzung im Merkur. Nach der historischen Zäsur vom 13. August 1961 versah er den Text mit dem Titelzusatz, veraltet', um zu markieren, dass sich mit den historischen auch die poetologischen Grundlagen grundlegend verändert hätten. Demzufolge lassen sich Johnsons Überlegungen vor allem auf die Arbeit an seinen ersten drei Romanen, Ingrid Babendererde, Mutmassungen über Jakob und Das dritte Buch über Achim zurückführen.
} 
Sucht man nach möglichen Vorbildern für Johnsons Poetologie, die „schwierige Suche nach der Wahrheit" ausdrücklich vorzuführen und damit auch formal abzubilden, erteilt der Autor dem Versuch höchstselbst eine Absage: „Ich habe keine persönlichen Vorbilder, und literarische Vorbilder gibt es für mich nicht. Jeder Schriftsteller muß sich seinen Stoff selber beschaffen, er muß sich seine Form selbst erarbeiten“ (zit. n. Schwarz 1988 [1969], S. 237). Im Sinne eines Vorrangs des Inhalts vor der Form, und Johnson war ein Autor, der in seiner Aktualität wie kaum ein anderer die jeweiligen zeitgeschichtlichen Themen zur Grundlage seiner Geschichten machte, ist diese Aussage überaus konsequent. Doch auch wenn ihm kein Autor als ein umfängliches Vorbild gedient haben mag - ein Umstand, den Johnson „,im Falle eines wirklichen Schriftstellers für ausgeschlossen“ halte -, eignete er sich das „Handwerkszeug früherer Schriftsteller“ an (Schwarz 1988 [1969], S. 237; Hervorhebung P.O.).

Nach der Fertigstellung seines Erstlings Ingrid Babendererde ${ }^{23}$ arbeitete Johnson seit dem Herbst 1956 an den Mutmassungen über Jakob. Begleitet wurde die Entstehung seines zweiten Romans durch einen intensiven Austausch über narratologische Themen wie die Zeitstruktur und die Stellung des Erzählers im Werk sowie die Perspektivierung des Erzählten vor dem Hintergrund des eigenen Germanistikstudiums, vor allem aber der Lektüre von zeitgenössischen Romanen und Erzählungen. Johnsons Gesprächspartner waren insbesondere seine Kommilitonen Manfred Bierwisch, Joachim Menzhausen und Klaus Baumgärtner, die er nach seinem Wechsel von der Universität Rostock an die Karl-Marx-Universität Leipzig im Herbst 1954 kennenlernte. ${ }^{24}$ Im Mittelpunkt dieses Austauschs stehen zwei der bedeutendsten Romanciers des 20. Jahrhunderts, die das Handwerkszeug des jungen Autors maßgeblich prägten: William Faulkner und James Joyce. $^{25}$

In seinen Erinnerungen Uwe Johnson betreffend erwähnt Manfred Bierwisch eine zentrale Lektüreerfahrung der Leipziger Freunde für den Sommer 1957. Johnsons Arbeit an den Mutmassungen über Jakob, insbesondere ihre Form

\footnotetext{
${ }^{23}$ Die letzten Versuche, den Roman zu veröffentlichen, scheiterten im Sommer 1957 durch Ablehnungen des Mitteldeutschen Verlags und des Suhrkamp Verlags (vgl. etwa Johnson 1980, S. 93-99).

${ }^{24}$ Zur als ,Leipziger Freunde " in die Johnson-Forschung eingegangenen Gruppe, deren Freundschaften weit über die Studienzeit hinaus anhielten, vgl. insbesondere Pautzke $(2013,2014)$.

${ }^{25}$ Neben Faulkner und Joyce finden sich in den Briefen der ,Leipziger Freunde' vor allem Hinweise auf Ernest Hemingway und die Stellung des Erzählers in dessen Romanen. Bernd Neumann (1993, S. 147) geht zudem davon aus, dass Johnson Theodor W. Adornos Vortrag Standort des Erzählers im zeitgenössischen Roman, der 1954 erschien, gekannt habe. Direkte Hinweise auf Adornos Vortrag finden sich allerdings weder in den Briefen der Freunde noch in Johnsons poetologischen Texten. An keiner Stelle greift er etwa Adornos Vergleich der Stellung des Erzählers im traditionellen Roman zur „Guckkastenbühne des bürgerlichen Theaters“ auf, sondern wählt für die Beschreibung des auktorialen Überblicks das Bild eines Tennisschiedsrichters (Adorno 1996 [1954], S. 45). Vgl. oben das ausführliche Zitat aus Johnson (1975 [1961]).
} 
betreffend, habe diese Erfahrung nachhaltig beeinflusst, denn zwischen einer ersten, linearen Fassung des Romans, einem ,ungebrochene[n] Stück Prosa in der geraden Erzählweise“, und der endgültigen Fassung in gebrochener Form, „die die Vorgänge auf die Perspektiven der Personen verteilt und diesen auch die Architektur des Zeitablaufs abgewinnt, liegt jenes exorbitante Zusammentreffen mit Faulkner [...]. Erst aus dem Ergebnis dieser Begegnung konnte auch der Titel hervorgehen, der wie ein Programm das Buch kennzeichnet“" (Bierwisch 1993, S. 85). ${ }^{26}$

Antje Pautzke hat die Korrespondenz der Leipziger Freunde hinsichtlich deren Lektüre von Faulkners Romanen wie Satoris (1929; dt. 1961), The Sound and the Fury (1929; dt. 1956), Light in August (1932; dt. 1935) oder Absalom, Absalom! (1936; dt. 1938) eingehend analysiert und gelangt zu dem Schluss, dass in den Briefen nicht nur offenbar werde, ,dass und wie Uwe Johnson die kanonischen Texte der Klassischen Moderne gelesen hat, sondern auch, dass und wie seine eigene Poetologie [...] durch den Austausch über die Lektüre von Autoren wie William Faulkner, aber auch James Joyce und Ernest Hemingway geformt worden ist“" (Pautzke 2014, S. 142). Veranschaulichen lässt sich Pautzkes These an einem Brief Johnsons an Klaus Baumgärtner vom 10. Dezember 1957, in dem er seine Eindrücke der Lektüre von Faulkners Satoris schildert:

Die Verleugnung der Chronologie, des zeitlichen Nacheinander schaffen ja eine Unzahl akzentuierter Höhen, wenn die einzelnen Vorfälle mit Zufälligkeit in der Erzählzeit verschränkt sind [...]. Nun bewegt $F$ sich ja immer rund um die Familie Sartoris, und nach seiner Technik bietet immer das Gemüt eines Familienmitglieds verschränkt mit späteren Nachrichten Entdeckungen etc. das Zentrum der Erzählung, für den Erzähler bleibt vorgeblich nichts als das mediale Arrangement. ${ }^{27}$

Bereits anhand dieses kurzen Briefausschnitts wird deutlich, dass Johnsons Augenmerk auf dem analytischen Erzählen Faulkners mit einer anachronistischen Darstellung der Geschichte um die Südstaatenfamilie Sartoris liegt. Auch in weiteren Briefen diskutieren die Leipziger Freunde insbesondere die Zeitstruktur in den Romanen Faulkners. Noch im März 1958 schreibt Bierwisch nach der Lektüre von Light in August an Johnson:

Beim Lesen von Faulkner geschrieben: Ob er, wenn das nicht zu schematisch wäre, nicht am liebsten seine Bücher rückwärts schreiben würde? Im Licht im August sieht dieser Zug so aus, daß er immer wieder, erst fürs ganze Buch, dann immer wieder unterteilend, das Ende eines Vorgangs zuerst berichtet, und dann erst sein Zustandekommen, ihn nun gleichsam begründend. Ohne daß es mit Exaktheit betrieben würde, entsteht dadurch das zwingende Bild von Vorgängen, die Stück für Stück ihre kausale Ursache haben: sie wird ja fast stets anschließend erzählt.

\footnotetext{
${ }^{26}$ Entsprechend bezeichnet Bernd Neumann (1993, S. 149) die umgearbeitete Fassung der Mutmassungen über Jakob als ,polyperspektivische, , verfaulknerte“ Form“.

${ }^{27}$ Uwe Johnson an Klaus Baumgärtner, 10.12.1957 zit. n. Pautzke (2014, S. 140), geprüft im Uwe Johnson-Archiv Rostock (Depositum der Johannes und Annitta Fries Stiftung).
} 
Dabei geschieht aber mit der Zeit, mit dem Zeitablauf in diesem Buch eine Umkehrung. Für The Sound And The Fury hat Sartre erläutert, daß Faulkners Zeitablauf keine Zukunftsdimension habe. Dies gilt ungefähr so auch hier. Vielleicht sagt man auch besser, es entsteht der Eindruck einer rückwärtsrollenden Zeit. Es ist aber damit notwendig etwas anderes verbunden (vielleicht ist es die Ursache dafür, daß die Zeit umgekehrt werden muß): Das begründende Erzählverfahren richtet den Blick vom Ereignis auf seine Vorgeschichte. ${ }^{28}$

Neben der analytischen Erzählweise, die für die von Bierwisch angeführten Romane Faulkners wie auch für die Mutmassungen über Jakob konstitutiv ist, geht Johnson im zuvor zitierten Brief an Baumgärtner auf die Rolle des Erzählers ein. Diesem bleibe ,vorgeblich nichts als das mediale Arrangement“ (s. o. Anm. 27). Ute Müller (2005, S. 258) führt daher Johnsons „oft belächeltes Beharren auf der ,realen“ Seinsweise seiner Figuren“ auf die Werke Faulkners zurück. Ähnlich wie Johnson darauf insistierte, dass seine Figuren ihm gegenüber ,unabhängige Personen“ (zit. n. Schwarz 1988 [1969], S. 239) ${ }^{29}$ seien, antwortete Faulkner im Mai 1957 im Rahmen eines Oberseminars über den amerikanischen Roman auf die Frage, inwieweit seine „Gestalten gewissermaßen ein Eigenleben“ besäßen und der Autor/Erzähler nur mehr als das „Werkzeug einer Idee“ betrachtet werden könne:

Wenn die Gestalten erst einmal zum Leben erweckt werden, dann begannen sie sich in der Tat zuweilen selbständig zu machen, und der Schriftsteller muß hinter ihnen herrennen und zur rechten Zeit aufzuschreiben versuchen, was sie sagen oder tun, und in diesem Sinne ist er tatsächlich ein Werkzeug. Die Gestalten haben dann die Führung übernommen, und von diesem Zeitpunkt an sind sie es, die die Geschichte erzählen. (zit. n. Gwynn und Blotner 1961, S. 149) ${ }^{30}$

Fragen zur Stellung des Erzählers zu den Figuren und zum Geschehen, auf denen das Spiel mit der (Un-)Zuverlässigkeit in den Mutmassungen über Jakob gründet, diskutieren die Leipziger Freunde aber insbesondere am Werk eines anderen Autors. Ende Juni 1957 vermeldet Bierwisch seinem Freund Johnson, dass er das erste Drittel von James Joyce Ulysses gelesen habe. ${ }^{31}$ Bierwisch fühlt sich durch die Lektüre dazu angeregt, grundsätzliche Überlegungen über die im Roman anzutreffenden inneren Monologe anzustellen und eine mögliche Polyperspektivität durch den Einsatz dieses Erzählmodus zu diskutieren. Johnson geht in seinem Antwortbrief auf die Erwägungen seines Freundes ein und erläutert darin seine poetologischen Vorstellungen, die für die narrative Form der Mutmassungen über Jakob grundlegend sind:

\footnotetext{
${ }^{28}$ Manfred Bierwisch an Uwe Johnson, 02.03.1958 zit. n. Pautzke (2014, S. 136 f.), geprüft im Uwe Johnson-Archiv Rostock.

${ }^{29}$ Vgl. hierzu etwa Göritz (1999, S. 46).

${ }^{30}$ Johnson besaß in seiner Privatbibliothek ein Exemplar dieses Bandes, der jedoch erst zwei Jahre nach Erscheinen der Mutmassungen über Jakob herausgegeben wurde. Vgl. Uwe JohnsonArchiv Rostock, UJA/BP/00722.

${ }^{31} \mathrm{Vgl}$. Manfred Bierwisch an Uwe Johnson, 23.06.1957, in: Uwe Johnson-Archiv Rostock, UJA/H/060044, B1. 13v.
} 
Was den „Ulysses“ angeht und den Wechsel der erzählenden Optik, so habe ich das schon verschiedentlich erwogen letztens als einen Grundsatz. In einer Beratung mit meiner privaten Leihbibliothek kamen wir überein: wenn Lieschen Müller ins Zimmer tritt und sich aufs Kanapee wirft und bitterlich weint, so ist noch lange nicht raus woher man das weiss. Es gebe ja nun drei Grundtypen des erzählenden Es: den Gott Balzac (man kann auch sagen: Schiedsrichter beim Tennis, denn der sitzt ja auch höher und weiss über alles); den Briefwechsel; die naturalistische (unterstellte) Beteiligung. Und die letzte ist gebräuchlich Filmes wegen und wegen Hemingway. Der mangelt der Überblick, die muss die Methode wechseln. Sternchen sind eine Erfreuung für Auge und Sinn des Lesers: meine ich, und: dass der Streit um die Optik in jenem Buch am zulänglichsten gelöst ist, das ist ein ästhetischer Fortschritt und so zu sagen eine Errungenschaft. ${ }^{32}$

Inwieweit sich Johnsons Überlegungen zum „Wechsel der erzählenden Optik“, die sich wenige Jahre später im Aufsatz Berliner Stadtbahn (veraltet) wiederfinden, unmittelbar auf Joyce und die eigene Lektüre des Ulysses zurückverfolgen lassen, geht aus dem Brief nicht hervor. Das Experimentieren mit der Erzählinstanz in seinem Erstling Ingrid Babendererde, ${ }^{33}$ vor allem aber die von Johnson mehrfach erwähnte erzählerische Umgestaltung der Mutmassungen über Jakob, die von Bierwisch bestätigt und auf den Sommer 1957 datiert wird, deuten auf den Einfluss des Ulysses und der Gespräche mit den Leipziger Freunden über den Roman auf Johnsons poetologische Erwägungen hin. Entsprechend konstatiert Maren Jäger (2009, S. 200), dass im dritten Typ, einem scheinbar am Erzählgeschehen beteiligten nichtdiegetischen Erzähler, die „,narrative Anlage der ,Mutmassungen“ vorweggenommen“ sei. Aus den Schwierigkeiten, die diese Erzählperspektive mit sich bringt, entwickelt Johnson für seinen zweiten Roman die Verteilung der narrativen Präsentation auf drei Erzählmodi; ein Erzähl(er)konzept, für das ,-- neben Faulkners ,The Sound and the Fury“ - die Polyphonie des ,Ulysses“ mit seinen wechselnden narrativen Mustern stilbildend“" war (Jäger 2009, S. 201).

Wie kontrovers die Leipziger Freunde vor dem Hintergrund von Johnsons eigenem Schreiben über diese und ähnliche narratologische Probleme diskutierten, verdeutlicht die Antwort Bierwischs, der sich mit der „Leihbibliothek“ seines Freundes „,nicht einverstanden“ erklärt: „Entweder man hält sich bei der Grundtatsache auf, daß jede: jede: Geschichte einen Erzähler hat und sich nicht selbst erzählt, also unabdingbar an irgend einer Stelle den Sprung tun muß zur Imagination. Insofern sind alle Techniken gleichen Wesens, ob Gottoder Wanzenperspektive. "34 Für die Frage nach dem unzuverlässigen Erzählen in den Mutmassungen über Jakob ist der übernächste Brief Bierwischs an Johnson

\footnotetext{
${ }^{32}$ Uwe Johnson an Manfred Bierwisch, 07.07.1957 zit. n. Jäger (2009, S. 200), geprüft im Uwe Johnson-Archiv Rostock.

${ }^{33}$ Vgl. hierzu etwa Leuchtenberger (2003, S. 92 f.) und Jäger (2009, S. 192 f.). Jäger vermutet, dass Johnson den Ulysses (erstmals) zwischen 1953 und 1957 gelesen habe (vgl. Jäger 2009, S. 184, 189).

${ }^{34}$ Manfred Bierwisch an Uwe Johnson, 13.07.1957 zit. nach Jäger (2009, S. 201 f.), geprüft im Uwe Johnson-Archiv Rostock.
} 
vom 4. August 1957 überaus aufschlussreich. Ein weiteres Mal auf die Erzählperspektive eingehend, bemerkt er:

Den Erzähler kann man entbehren, sagst Du, weil der Bewußtseinsgrad der Gestalten so angewachsen ist, daß aus ihrem Horizont heraus alles gesagt werden kann. Der Schiedsrichter wird arbeitslos.

Das ist (sehen wir von Doktor Faustus ab, der sich in Zeitbloom eine eigene Form von Erzähler zurecht macht) ein ausgesprochener Trugschluß. Denn wie kommt es, daß einer der ganz reale Erzähler - das Bewußtsein von x Personen kennt und darlegen kann? Wenn eine Geschichte, ganz immanent, die Gedanken von A und dann von B darlegt, hat sie damit schon den Erzähler zu erkennen gegeben. Beachte das bitte gebührend. Denn ohne Fiktion kann nur der Inhalt eines Bewußtseins aufgeschrieben werden. (Das hieße, daß alle Personen aufs wirklich Erfahrbare, ihre Rede und Taten beschränkt würden, womit aus der Erzählung Drama würde. Und dem nähern sich ja scheinbar die Dialoge Hemingways an. Aber eben nur scheinbar.)

Um also den Erzähler-Gott auszuschalten, müßte man sich auf die Ich-Form beschränken, oder alles nicht wirklich Erfahrbare aussparen - und sich dem Drama nähern, das wirklich keinen Erzähler hat (wenn er nicht von Brecht wieder hineingebracht worden wäre.) Aber selbst das ist dann nur scheinbar ein Bericht ohne Schiedsrichter. ${ }^{35}$

Bierwisch weist die Vorstellung seines Freundes, durch die figurale und damit polyperspektivische Präsentation von Bewusstseinsvorgängen könne auf einen (auktorialen) ,Erzähler-Gott' verzichtet werden, als eine Scheinlösung, einen Trugschluss zurück. Als neuralgischen Punkt der Diskussion um die Erzählperspektive macht er die parallele Wiedergabe der Gedanken und Gefühle mehrerer Figuren in einem Werk aus - ein Punkt, der in den Mutmassungen über Jakob von essenzieller Bedeutung und in der vorliegenden Untersuchung zur (Un-)Zuverlässigkeit des Erzählers insofern relevant ist, als der Erzähler scheinbar über das Bewusstsein einer bereits verstorbenen Figur zu verfügen vermag. Einzig durch die Integration einer auktorialen, nichtdiegetischen Erzählinstanz könne das Bewusstsein mehrerer Figuren (in zuverlässiger Weise) wiedergegeben werden. Mit dem Verzicht auf einen nichtdiegetischen Erzähler gehe zugleich eine Beschränkung der Möglichkeiten der Bewusstseinswiedergabe einher: Mittels eines diegetischen (Ich-)Erzählers oder im dramatischen Modus könne das Bewusstsein nur (jeweils) einer Figur wiedergegeben werden. Einen Ausweg aus dem Kreis der Erzählperspektive hält Bierwisch für ausgeschlossen:

es kann da auch keinen Fortschritt geben. Nur der Bruch kann an einer anderen Stelle liegen. (Darum gibt es, trotz aller aller Experimente, in der Epik nicht einen Bruch der Art am Anfang der Moderne, wie andere Künste ihn spüren lassen, von der Malerei bis zur Lyrik.

Oder vielmehr: die Versuche, diesen Kreis zu sprengen, sind als Erschütterungen andrer Art zu spüren. Nur eben: Der Erzähler ist nicht totzuschlagen.) $)^{36}$

\footnotetext{
${ }^{35}$ Manfred Bierwisch an Uwe Johnson, 04.08.1957, in: Uwe Johnson-Archiv Rostock, UJA/H/060048, Bl. 20-22v, hier: B1. 21-21v.

${ }^{36}$ Uwe Johnson-Archiv Rostock, UJA/H/060048, B1. 22.
} 
Dass Johnson die Kritik seines Freundes ernst nahm, lässt sich einerseits an der Konzeption seines zweiten Romans nachverfolgen, andererseits an seinem poetologischen Aufsatz Berliner Stadtbahn (veraltet), in dem er eingesteht, dass bei der schwierigen Suche nach der Wahrheit Gesten des Erzählens entstünden, „deren epischer Charakter umstritten ist, aber wenn zum Beispiel mit den erzählten Vorfällen wirksam verbunden ein ideologisches System vorkommt, so scheint dessen Diskussion auch eine Weise davon zu erzählen und nicht die am meisten unhandliche“" (Johnson 1975 [1961], S. 20 f.).

Eine Erschütterung, wie sie Bierwisch beschreibt, erfährt das Erzählen in den Mutmassungen über Jakob durch einen Erzähler, der in seiner Erzählperspektive changiert. Einer bewussten Zurückhaltung, die die Perspektive der Figuren in ihrer Geltung stärkt, stehen Momente gegenüber, in denen der Erzähler scheinbar in der Lage ist, auf das Bewusstsein der Figuren zurückzugreifen - gar auf das des zu Beginn des Erzählens bereits verstorbenen Jakob. Dass es sich bei der Wiedergabe der Gedanken und Gefühle der Titelfigur (mutmaßlich) um szenische Vergegenwärtigungen handelt - ein Kunstgriff, den Ute Müller (2005, S. 272) u. a. auf Johnsons Lektüre von William Faulkners Romanen zurückführt - konnte anhand von Indizien innerhalb der Erzählerpartien erschlossen werden. Offenbar ganz bewusst nimmt Johnson eine gewisse mimetische Unzuverlässigkeit der Erzählerpartien in Kauf, um einen Erzähler zu konstituieren, der gar nicht erst versucht, in einem gottgleichen Überblick eine Wahrheit zu präsentieren. Vielmehr führt der Erzähler, den Johnson als ,,jemanden“ verstanden wissen möchte, „der auch von dem Unglück gehört hat, und der auch nur wenig mehr von den zugehörigen Ereignissen weiß oder sie auch erzählt bekommen hat“" (zit. n. Roloff 1988 [1961], S. 177), ${ }^{37}$ durch das latente Spiel mit der mimetischen (Un-)Zuverlässigkeit die schwierige Suche nach der Wahrheit angesichts eines nebulös bleibenden Todesfalls aus möglichst vielen verschiedenen Blickwinkeln vor.

\section{Literatur}

Adorno, Theodor W.: Standort des Erzählers im zeitgenössischen Roman [1954]. In: ders.: Gesammelte Schriften, hg. von Rolf Tiedemann, Bd. 2: Noten zur Literatur, 4. Aufl., Frankfurt am Main 1996, 41-48.

Ahl, Herbert: Im Westen fremd - im Osten heimatlos. In: Diplomatischer Kurier 9, 1960, H. 4, $149-152$.

Bierwisch, Manfred: Erinnerungen Uwe Johnson betreffend. In: Roland Berbig/Erdmut Wizisla: „Wo ich her bin ... “. Uwe Johnson in der DDR, Berlin 1993, 80-91.

Blöcker, Günter: Roman der beiden Deutschland. In: Frankfurter Allgemeine Zeitung, Nr. 253 vom 31. Oktober 1959, Bilder und Zeiten, V.

Bond, Greg: „Die Grossen des Landes warfen ein Auge auf Jakob“. Uwe Johnsons Mutmassungen über Jakob. In: treibhaus 5: Das Jahr 1959 in der deutschsprachigen Literatur, 2009, 144-159.

\footnotetext{
${ }^{37}$ Vgl. hierzu Neumann (1993, S. 154-156).
} 
Born, Arne: Wie Uwe Johnson erzählt. Artistik und Realismus des Frühwerks, Hannover 1997.

Fahlke, Eberhard: Die „Wirklichkeit“ der Mutmassungen. Eine politische Lesart der Mutmassungen über Jakob von Uwe Johnson, Frankfurt am Main 1982.

Genette, Gérard: Die Erzählung, aus dem Französischen von Andreas Knop, mit einem Nachwort hg. von Jürgen Vogt, München 1994 [1972].

Göritz, Matthias: Die Ethik des Geschichtenerzählens in Uwe Johnsons Mutmassungen über Jakob. In: Johnson-Jahrbuch 6, 1999, S. 38-56.

Gwynn, Frederick L./Blotner, Joseph L.: Gespräche mit Faulkner, mit einer Einleitung von Erich Franzen, Deutsch von Helmut Hilzheimer, Stuttgart 1961.

Halstenberg, Armin: „Dichter sollte man nicht stören“. Heute am Telefon: Uwe Johnson (Am 19.7.1969 in West-Berlin). In: Eberhard Fahlke (Hg.), „Ich überlege mir die Geschichte“. Uwe Johnson im Gespräch, Frankfurt am Main 1988, S. 231-233.

Jäger, Maren: Die Joyce-Rezeption in der deutschsprachigen Erzählliteratur nach 1945, Tübingen 2009.

Johnson, Uwe: Mutmassungen über Jakob, Historisch-kritische Ausgabe der Werke, Schriften und Briefe Uwe Johnsons (Rostocker Ausgabe), Abt. I: Werke, Bd. 2, hg. von Astrid Köhler u. a., Frankfurt am Main 2017 [1959].

Uwe Johnson: Berliner Stadtbahn (veraltet) [1961]. In: ders.: Berliner Sachen. Aufsätze, Frankfurt am Main 1975, S. 7-21.

Johnson, Uwe: Wenn Sie mich fragen ... (Ein Vortrag) [1975]. In: Eberhard Fahlke (Hg.), „Ich überlege mir die Geschichte“. Uwe Johnson im Gespräch, Frankfurt am Main 1988, 51-64.

Johnson, Uwe: Begleitumstände. Frankfurter Vorlesungen, Frankfurt am Main 1980.

Kindt, Tom: Unzuverlässiges Erzählen und literarische Moderne. Eine Untersuchung der Romane von Ernst Weiß, Tübingen 2008.

Krellner, Ulrich: „Was ich im Gedächtnis ertrage“. Untersuchungen zum Erinnerungskonzept von Uwe Johnsons Erzählwerk, Würzburg 2003.

Lang, Simone Elisabeth: „Zumindest hätte er sich all das gewünscht. “ Unzuverlässiges Erzählen in der Heterodiegese, Diss. Bremen 2016.

Leuchtenberger, Katja: „,Wer erzählt, muß an alles denken“. Erzählstrukturen und Strategien der Leserlenkung in den frühen Romanen Uwe Johnsons, Göttingen 2003.

Mayer, Hans: „Mutmassungen über Jakob“. In: Raimund Fellinger (Hg.): Über Uwe Johnson, Frankfurt am Main 1992, S. 85-94.

Müller, Ute: William Faulkner und die Deutsche Nachkriegsliteratur, Würzburg 2005.

Neumann, Bernd: Vexierrätsel, Gesamtdeutsch. Über die Ursprünge von Uwe Johnsons Debütroman Mutmassungen über Jakob. In: Interpretationen. Romane des 20. Jahrhunderts, Bd. 2, Stuttgart 1993, 143-185.

Neusüss, Arnhelm: Über die Schwierigkeiten beim Schreiben der Wahrheit. Gespräch mit Uwe Johnson (Am 10.9.1961 in West-Berlin). In: Eberhard Fahlke (Hg.), „Ich überlege mir die Geschichte“. Uwe Johnson im Gespräch, Frankfurt am Main 1988, 184-193.

Pautzke, Antje: Der Literat und der Zeitzeuge. Zwei Arten, in Uwe Johnsons Briefen zu lesen. In: Johnson-Jahrbuch 20, 2013, 209-223.

Pautzke, Antje: Uwe Johnson liest. William Faulkner im Briefwechsel der Leipziger Freunde. In: Johnson-Jahrbuch 21, 2014, S. 131-142.

Phelan, James/Martin, Mary Patricia: The Lessons of „Weymouth“. Homodiegesis, Unreliability, Ethics and The Remains of the Day. In: David Herman (Hg.): Narratologies. New Perspectives on Narrative Analyses, Columbus 1999, 88-109.

Reich-Ranicki, Marcel: Ein Eisenbahner aus der DDR. Zu Uwe Johnsons Roman „Mutmaßungen über Jakob“. In: Das Sonntagsblatt, Nr. 47 vom 22.11.1959, 20.

Roloff, Michael: Gespräch mit Uwe Johnson (Am 20.8.1961 in New York). In: Eberhard Fahlke (Hg.): „Ich überlege mir die Geschichte“. Uwe Johnson im Gespräch, Frankfurt am Main 1988, 171-183.

Sacharov, Jurij: Wiederholungen in Uwe Johnsons Roman Mutmassungen über Jakob im Kontext der erzählerischen Kompetenz des Narrators. In: Bohdan Maxymtschuk/Stefan J. Schier- 
holz (Hg.): Die Lemberger Germanistik in der Ukraine. Innensichten - Außensichten - Dissertationsvorhaben, Frankfurt am Main 2011, 79-88.

Schmid, Wolf: Elemente der Narratologie, 2., verb. Aufl., Berlin 2008.

Schwarz, Wilhelm J.: Gespräche mit Uwe Johnson (Am 10.7.1969 in West-Berlin). In: Eberhard Fahlke (Hg.): „Ich überlege mir die Geschichte“. Uwe Johnson im Gespräch, Frankfurt am Main 1988, 234-247

Wieser, Theodor: „Mutmaßungen über Jakob“. Roman von Uwe Johnson. In: Neue Zürcher Zeitung (Morgenausgabe), Nr. 3789 vom 5.12.1959, 25.

Yamamoto, Hiroshi: „Über das Gewicht des Formalen“ stolpernd. Zur zögerlichen JohnsonRezeption in Japan. In: Johnson-Jahrbuch 22, 2015, 59-73.

Open Access Dieses Kapitel wird unter der Creative Commons Namensnennung 4.0 International Lizenz (http://creativecommons.org/licenses/by/4.0/deed.de) veröffentlicht, welche die Nutzung, Vervielfältigung, Bearbeitung, Verbreitung und Wiedergabe in jeglichem Medium und Format erlaubt, sofern Sie den/die ursprünglichen Autor(en) und die Quelle ordnungsgemäß nennen, einen Link zur Creative Commons Lizenz beifügen und angeben, ob Änderungen vorgenommen wurden.

Die in diesem Kapitel enthaltenen Bilder und sonstiges Drittmaterial unterliegen ebenfalls der genannten Creative Commons Lizenz, sofern sich aus der Abbildungslegende nichts anderes ergibt. Sofern das betreffende Material nicht unter der genannten Creative Commons Lizenz steht und die betreffende Handlung nicht nach gesetzlichen Vorschriften erlaubt ist, ist für die oben aufgeführten Weiterverwendungen des Materials die Einwilligung des jeweiligen Rechteinhabers einzuholen. 